\title{
Anesthetic management of down syndrome patients in surgical procedures
}

\begin{abstract}
Patients with Down syndrome may have significant cardiovascular, respiratory, central nervous, skeletal, endocrine, gastrointestinal, and others system anomalies, most of which require early surgical intervention. Anesthetic management of the patients with Down syndrome should be required carefully preoperative evaluation, closely intraoperative and postoperative monitoring in the surgical procedures.
\end{abstract}

Keywords: down syndrome, anesthesia, management
Volume II Issue 3 - 2019

\author{
Ahmet Eroglu \\ Karadeniz Technical University, Anesthesiology and Intensive \\ Care Medicine, Turkey
}

\begin{abstract}
Correspondence: Ahmet Eroglu, Karadeniz Technical
University, Anesthesiology and Intensive Care Medicine, Trabzon,

Turkey,Emaileroglua@hotmail.com
\end{abstract}

Received: December 19, 2019 | Published: December 26,

2019

\section{Introduction}

Down syndrome is the most common chromosome disorder affecting all body systems in live born infants, and It is caused by the inheritance of an additional chromosome 21. ${ }^{1}$ Patients with Down syndrome may have significant cardiovascular, respiratory, central nervous, skeletal, endocrine, gastrointestinal, and others system anomalies, most of which require early surgical intervention. A safe anesthesia or sedoanalgesia should perform before, during and after the surgical procedures. The aim of this report is to evaluate the anesthetic management of the patients with Down syndrome in surgical procedures. A history and examination of the patient is required to identify the problems of all systems prior to anesthesia. Acyanotic congenital heart defects such atrioventricular septal defect (AVSD), ventricular septal defect (VSD), patent ductus arteriosus (PDA), atrial septal defect (ASD) and cyanotic heart defects should be determined. If there is an acynotic heart defect increases in peripheral vascular resistance and high SVR can be avoid during the procedure. Treatment of intraopertaive Tetralogy of Fallot should be managed with fluid, oxygen and SVR must be keep within normal limits.

Airway and pulmonary system of the patient should be evaluated related to macroglossia, enlarged tongue, tonsils and adenoids, subglottic stenosis, obstructive sleep apnea (OSA) and other airway obstructions. Atlanto-axial instability should be evaluated with a radiograph of the cervical spine. If difficult peripheral venous access is present, topical local anesthetic may be applied to facilitate intravenous cannulation. Oral or parenterally midazolam may be used for premedication. Premedication should be used cautiously in children with severe OSA. Required preparations must be done for difficult airway and intubation with advanced airway equipment. A smaller tracheal tube may be needed due to subglottic stenosis. If gastrooesophageal reflux risk is present, premedication with an H2 antagonist may be beneficial. Patients with Down syndrome must be evaluated for hypothyroidism before anesthesia. Antibiotic prophylaxis should be given for infective endocarditis in children with structural heart lesions.

Standard monitoring including ECG, pulseoximetry, end tidal $\mathrm{CO}_{2}$ monitor, blood pressure and temperature monitor should be used in all cases. Anesthesia may induce and maintain with inhaler, total intravenous or regional block in specific patients. Patients should be observed closely in the postanesthetic care unit (PACU) until full recovery from anesthesia after the surgical procedure. Adults with Down syndrome need to undergo regular medical examinations, and Laegefoen and colleagues ${ }^{2}$ recommend an annual check-up with the primary doctor.

\section{Conclusion}

Anesthetic management of the patients with Down syndrome should be required carefully preoperative evaluation, closely intraoperative and postoperative monitoring in the surgical procedures.

\section{Conflicts of interest}

I have no conflict of interest in relation to this short communication.

\section{References}

1. Lewanda AF, Matisoff A2, Revenis M, et al. Preoperative evaluation and comprehensive risk assessment for children with Down syndrome. Paediatr Anaesth. 2016;26(4):356-362.

2. Malt EA, Dahl RC, Haugsand TM, et al. Health and disease in adults with Down syndrome. Tidsskr Nor Laegeforen. 2013;133: 290-294. 